\title{
The Potential Role of Gymnema inodorum Leaf Extract Treatment in Hematological Parameters in Mice Infected with Plasmodium berghei
}

\author{
Sakaewan Ounjaijean $\mathbb{D}^{1},{ }^{1}$ Suriyan Sukati, ${ }^{2}$ Voravuth Somsak $\mathbb{D}^{2,3}$ and Orawan Sarakul $\mathbb{D}^{2}$ \\ ${ }^{1}$ School of Health Sciences Research, Research Institute for Health Sciences, Chiang Mai University, Chiang Mai 50200, Thailand \\ ${ }^{2}$ School of Allied Health Sciences, Walailak University, Nakhon Si Thammarat 80161, Thailand \\ ${ }^{3}$ Research Excellence Center for Innovation and Health Products, Walailak University, Nakhon Si Thammarat 80161, Thailand
}

Correspondence should be addressed to Orawan Sarakul; sorawan@wu.ac.th

Received 25 March 2021; Accepted 19 June 2021; Published 29 June 2021

Academic Editor: Alemayehu Toma

Copyright (C) 2021 Sakaewan Ounjaijean et al. This is an open access article distributed under the Creative Commons Attribution License, which permits unrestricted use, distribution, and reproduction in any medium, provided the original work is properly cited.

\begin{abstract}
Malaria remains a significant cause of death in tropical and subtropical regions by serious complications with hematological abnormalities consistent with high parasitemia. Hence, this study aimed to determine the efficacy of the Gymnema inodorum leaf extract (GIE) on hematological alteration in Plasmodium berghei infection in mice. Groups of ICR mice were infected intraperitoneally with parasitized red blood cells of $P$. berghei ANKA (PbANKA). They were administered orally by gavage of 100,250 , and $500 \mathrm{mg} / \mathrm{kg}$ of GIE for 4 consecutive days. Healthy and untreated groups were given distilled water, while $10 \mathrm{mg} / \mathrm{kg}$ of chloroquine was treated as the positive control. Hematological parameters including RBC count, hemoglobin (Hb), hematocrit (Hct), mean corpuscular volume (MCV), mean cell hemoglobin $(\mathrm{MCH})$, mean cell hemoglobin concentration (MCHC), RBC distribution width (RDW), white blood cell (WBC) count, and WBC differential count were measured. The results showed that significant decreases of RBC count, $\mathrm{Hb}, \mathrm{Hct}, \mathrm{MCV}, \mathrm{MCH}, \mathrm{MCHC}$, and reticulocytes were observed in the untreated group, while RDW was significantly increased compared with the healthy control. Furthermore, the WBC, neutrophil, monocyte, basophil, and eosinophil of untreated mice increased significantly, while the lymphocyte was significantly decreased compared with the healthy control. Interestingly, GIE normalized the hematological alteration induced by PbANKA infection in GIE-treated groups compared with healthy and untreated groups. The highest efficacy of GIE was observed at a dose of $500 \mathrm{mg} / \mathrm{kg}$. Our results confirmed that GIE presented the potential role in the treatment of hematological alteration during malaria infection.
\end{abstract}

\section{Introduction}

Malaria remains a public health burden caused by a parasite in the genus Plasmodium and transmitted by the female Anopheles mosquito. Five species of Plasmodium are infectious to humans, including Plasmodium falciparum, Plasmodium vivax, Plasmodium malariae, Plasmodium ovale, and Plasmodium knowlesi [1]. It is estimated that $90 \%$ of malaria-related deaths occur in sub-Saharan Africa, most of which are children under five years old. The death due to malaria parasite infection is caused by critical complications such as cerebral malaria, severe hemolytic anemia, metabolic acidosis, respiratory distress, liver dysfunction, acute kidney injury, and hematological abnormalities [2]. Malaria-induced hematological abnormalities are also associated with hemoglobinopathy, nutritional status, and immunity $[3,4]$. However, several research studies revealed the increase in development and spread of antimalarial resistance by malaria parasites and toxicities associated with standard antimalarial drugs [5]. Hence, the search for alternatives, especially from plants that present an antimalarial and a wide range of biological activities, is urgently needed.

Gymnema inodorum (Lour.) Decne. (Phak Chiang Da as a local name) belongs to the Asclepiadaceae family and has been known to be effective for diseases, including diabetes mellitus, gout, and rheumatoid arthritis. This plant is 
indigenous in Southeast Asia including Thailand, particularly in the northern part of the country, and is widely consumed [6]. G. inodorum leaf extract showed high antioxidant and anti-inflammatory activities with polyphenols and flavonoids as the major compounds [7]. It has also been found that the leaf extract of $G$. inodorum could decrease blood glucose by inhibiting glucose absorption [8,9]. G. sylvestre is the same genus that is popular in India for suppressing glucose absorption and preventing type 2 diabetes mellitus $[10,11]$. The leaves of this plant also have polyphenols, flavonoids, triterpenoid saponins, anthraquinones, alkaloids, tannin, quinones, and gymnemic acids, which are the major active compounds of $G$. sylvestre leaf extract [12]. In vitro and in vivo studies of $G$. sylvestre leaf extract described the pharmacological properties, including antidiabetic, anticancer, antibacterial, antioxidant, anti-inflammatory, antimalarial, hepatoprotective, and immunosuppressive activities [13]. In addition, gymnemic acids and other constituents have been found in G. inodorum leaf extract, so this plant might have properties found in $G$. sylvestre. However, the studies regarding the hematological effect of $G$. inodorum in the rodent malaria model have never been carried. In malarial studies using rodent models, $P$. berghei is a suitable model due to the most common characteristics with $P$. falciparum [14]. Therefore, it is necessary to evaluate $G$. inodorum leaf extract's therapeutic potential on hematological abnormalities during $P$. berghei infection in mice.

\section{Materials and Methods}

2.1. Chemicals. Chloroquine diphosphate salt (CQ) purchased from Sigma (Sigma Chemical, St Louis, MO, USA) was used in the present study. All chemicals and reagents were of analytical grade and procured from the certificated suppliers.

2.2. Collection of Plant Material. The leaves of Gymnema inodorum was obtained from the Chiangda organic company garden (Chiang Mai, Thailand). This plant was identified correctly and authenticated by the plant biologists at Chiang Mai University. The voucher specimen of the plant was deposited in the Research Institute for Health Sciences, Chiang Mai University, Chiang Mai, Thailand.

\subsection{Preparation of the Aqueous Leaf Extract of Gymnema} inodorum. Fresh leaves of $G$. inodorum were washed with clean water and dried at $60^{\circ} \mathrm{C}$ in a hot air oven overnight. The dried leaves were ground into powder in a mortar with pestle and subsequently pulverized into fine powder using an electric blender. The powdered plant material was extracted in distilled water (DW) at a proportion of $5 \mathrm{~g} \%$. This mixture was allowed to incubate at $60^{\circ} \mathrm{C}$ in the incubator shaker at $300 \mathrm{rpm}$ for $30 \mathrm{~min}$. Centrifugation was carried out at $2,500 \mathrm{rpm}$ for $15 \mathrm{~min}$, and then the supernatant was collected. After filtration through Whatman no. 1 filter paper, the filtrate was lyophilized to dryness. The G. inodorum leaf extract (GIE) was stored at $-20^{\circ} \mathrm{C}$ before experiment [15].
2.4. Preparation of the Antimalarial Drug. CQ at a dose of $10 \mathrm{mg} / \mathrm{kg}$ was freshly prepared corresponding to the mouse's body weight in $0.3 \mathrm{ml}$ of DW and treated orally by gavage.

2.5. Animal Preparation. The experimental mice used in the present study were male ICR mice, aged about 4-6 weeks, weighing 25-30 g, and purchased from the Nomura Siam International Co., Ltd. They were housed in the animal room with temperature control $\left(25+0.5^{\circ} \mathrm{C}\right)$ and $12 \mathrm{~h}$ light- $12 \mathrm{~h}$ dark cycle. The mice were fed with the standard pellet diet (CP082) and clean drinking water, ad libitum. The experiments involving animals were carried out following the guideline for the care and use of laboratory animals and were approved by the animal care and use committee, Walailak University (WU-AICUC-63-031).

2.6. Plasmodium berghei. The rodent malaria parasite Plasmodium berghei strain ANKA (PbANKA) was obtained from the MR4 (Malaria Research and Reference Reagent Resource Center). The stock of parasitized red blood cells (RBC) of PbANKA was inoculated intraperitoneally into the donor naïve ICR mice. Parasitemia was monitored daily by microscopy of Giemsa-stained blood film. The mice parasitized with PbANKA recording 20-30\% parasitemia were then sacrificed, and blood was collected by cardiac puncture. Blood was diluted in phosphate buffer solution (PBS) to obtain $1 \times 107$ parasitized RBC in the volume of $0.3 \mathrm{ml}$, and the naive ICR mice were infected.

2.7. Parasitemia Determination. Blood was collected from the tail vein of each mouse and used to make a thin blood smear. The smear was fixed with absolute methanol and subsequently stained with $10 \%$ Giemsa for $10 \mathrm{~min}$. Percent parasitemia was determined by counting the number of parasitized RBC out of 2,000 RBC in random fields under a microscope with a $100 \mathrm{x}$ oil immersion lens. Percent parasitemia was calculated according to the following formula:

$$
\% \text { parasitemia }=\frac{\text { total number of parasitized } \mathrm{RBC} \times 100}{\text { total number of } \mathrm{RBC}} .
$$

2.8. Hematological Parameter Determination. Mice were sacrificed, and blood was collected by cardiac puncture into the EDTA vacuum tubes. Complete blood count (CBC) including RBC count, hemoglobin $(\mathrm{Hb})$, hematocrit $(\mathrm{Hct})$, mean corpuscular volume (MCV), mean cell hemoglobin $(\mathrm{MCH})$, mean cell hemoglobin concentration (MCHC), RBC distribution width (RDW), reticulocytes, white blood cell (WBC) count, and WBC differential was subsequently performed using the automate analyzer system at the Research Institute for Health Sciences, Walailak University. In addition, EDTA blood was also diluted with $1 \%$ brilliant cresyl blue in an equal volume. After mixing gently, incubation at $37^{\circ} \mathrm{C}$ for $15 \mathrm{~min}$ was performed. The smear was subsequently prepared, and reticulocytes were counted 
under a light microscope using a 100x oil immersion lens. Percent reticulocytes were calculated according to the following formula:

$$
\% \text { reticulocyte }=\frac{\text { total number of reticulocyte } \mathrm{RBC} \times 100}{\text { total number of } \mathrm{RBC}} .
$$

\subsection{Effect of GIE on Hematological Parameters in PbANKA- Infected Mice. Standard Peters' test was carried out to de- termine the effect of GIE on hematological parameters in mice infected with PbANKA [14]. Naïve ICR mice were divided into 6 groups of 3 mice each. Group I was neither infected with PbANKA nor treated with the extract as healthy control. Group II was infected with PbANKA but was not treated with the extract as the untreated control. Group III, IV, and V were infected with PbANKA and treated with 100,250 , and $500 \mathrm{mg} / \mathrm{kg}$ of GIE, respectively. Group VI was infected with PbANKA and treated with $10 \mathrm{mg} / \mathrm{kg}$ of CQ as a positive control. The treatment was administered orally by gavage once a day for 4 consecutive days (day 0-3). On day 4, the mice were sacrificed, and blood from each mouse was collected by cardiac puncture. Fresh blood was used to prepare a thin blood smear for parasitemia determination and the measurement of $\mathrm{CBC}$ using an au- tomated analyzer.}

2.10. Data Analysis. All statistical analyses were performed using the GraphPad Prism software (GraphPad Prism software, Inc., USA). The values were expressed as mean+ standard error of mean (SEM), and comparisons were made using one-way ANOVA with Tukey's post hoc test. Statistical significance was considered at $95 \%$ confidence, $p<0.05$.

\section{Results}

3.1. Hematological Alteration of RBC in GIE-Treated Mice. The results of hematological parameters of RBC are presented in Figure 1. PbANKA-infected mice in the untreated group induced significant $(p<0.05)$ decreases in $\mathrm{RBC}$ count, $\mathrm{Hb}, \mathrm{Hct}, \mathrm{MCV}, \mathrm{MCH}, \mathrm{MCHC}$, and reticulocytes, when compared with the healthy control (Figures $1(\mathrm{a})-1(\mathrm{f})$ and $1(\mathrm{~h}), \mathrm{UN})$. RDW was found to be significantly $(p<0.01)$ higher in the untreated mice than the healthy control. The treatment of infected mice with GIE at a dose of $500 \mathrm{mg} / \mathrm{kg}$ and CQ restored the altered hematological indices compared with the healthy control. There were significant $(p<0.05)$ differences when the hematological indices of the GIE- and CQ-treated groups were compared with those of the untreated mice (Figures 1(a)-1(h), GI500 and CQ). This indicated that the GIE normalized the hematological alteration of $\mathrm{RBC}$ generated by PbANKA infection. However, reticulocytes were significantly $(p<0.05)$ decreased in GIEtreated groups and increased for the CQ-treated group when compared with the healthy control (Figure 1(h), GI100, GI250, GI500, and CQ).
3.2. Hematological Alteration of WBC in GIE-Treated Mice. The alteration of hematological parameters of WBC in $\mathrm{PbANKA}$-infected mice treated with GIE is presented in Figure 2 . There was a significantly $(p<0.05)$ elevated WBC, neutrophil, monocyte, basophil, and eosinophil count, while a significant $(p<0.001)$ decrease in lymphocytes was observed in untreated groups compared with the healthy control (Figures 2(a)-2(f), UN). The GIE at a dose of $500 \mathrm{mg} / \mathrm{kg}$ and CQ normalized the hematological alteration of WBC during PbANKA infection in mice (Figures 2(a)2(e), GI500 and CQ). This implied that GIE restored WBC homeostasis in mice infected with PbANKA. However, eosinophils were significantly $(p<0.05)$ higher in infected mice treated with GIE compared with the healthy control (Figure 2(f), GI100, GI250, and GI500).

\section{Discussion}

In this study, GIE showed the ability to restore hematological parameters induced by $\mathrm{PbANKA}$ infection in mice. Hematological parameters of $\mathrm{RBC}$ and $\mathrm{WBC}$ are common biomarkers of malaria infection and indicators of compounds against malaria infection. The results showed that significant decreases in RBC count, $\mathrm{Hb}, \mathrm{Hct}, \mathrm{MCV}, \mathrm{MCH}$, $\mathrm{MCHC}$, and reticulocytes as well as increased RDW were observed in the untreated group compared with healthy control. This suggested that infected mice suffered from severe hemolysis and had hypochromic microcytic anemia during $\mathrm{PbANKA}$ infection $[3,16]$. It might be due to rapid $\mathrm{RBC}$ destruction and lysis of both parasitized and uninfected RBC, bone marrow and erythropoietic suppression, and dyserythropoiesis [17, 18]. In addition, it has also been reported that an increase in $\mathrm{RBC}$ fragility led to hemolysis followed by anemia in the PbANKA-infected mice [19]. Several studies have revealed that proinflammatory cytokines such as interferon- $\gamma$ (IFN- $\gamma$ ), tumor necrosis factor- $\alpha$ (TNF- $\alpha$ ), macrophage migration inhibitory factor, and hemozoin played an important role in the pathogenesis of hemolysis and anemia during malaria infection by erythropoiesis resistance, decreasing reticulocytosis, and inhibiting erythropoietin-induced erythroid precursor proliferation [20-23]. Moreover, malaria infection increased immunoglobulin-G autoantibody against uninfected RBC, decreasing RBC deformability and improving erythrophagocytosis [24]. According to our previous observation that GIE had no antimalarial activity (data not shown), the ability of GIE to restore RBC count, $\mathrm{Hb}, \mathrm{Hct}, \mathrm{MCV}, \mathrm{MCH}$, $\mathrm{MCHC}$, and RDW as well as significantly decrease reticulocytes when compared with the healthy and untreated groups suggested that the GIE might process the protective effect and immunomodulating and erythropoietic activities. These effects would be due to the presence of active metabolites in the GIE. The effect of GIE on hematological parameters of RBC was consistent with the standard antimalarial drug CQ. It was found that CQ-treated mice showed a significant increase in reticulocytes when compared with the healthy and untreated groups. It has been reported that CQ could result in increased erythropoietin levels in association with its anti-inflammation, resulting 


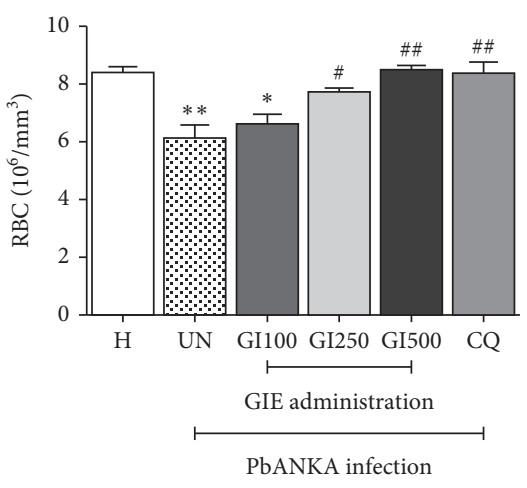

(a)

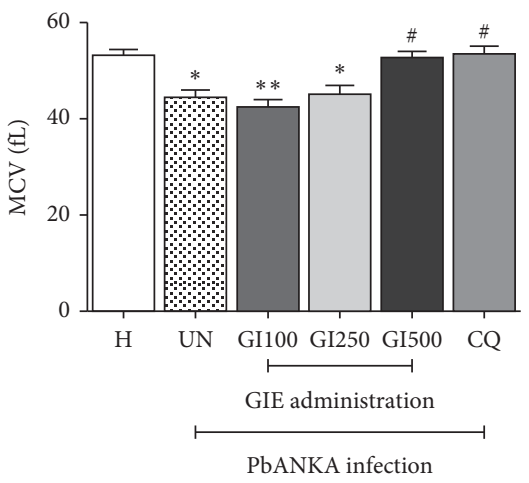

(d)

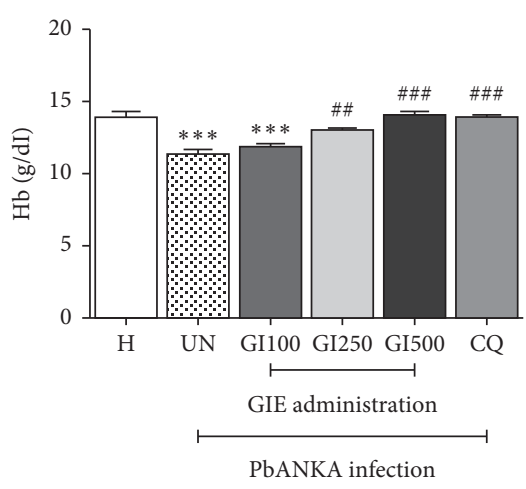

(b)

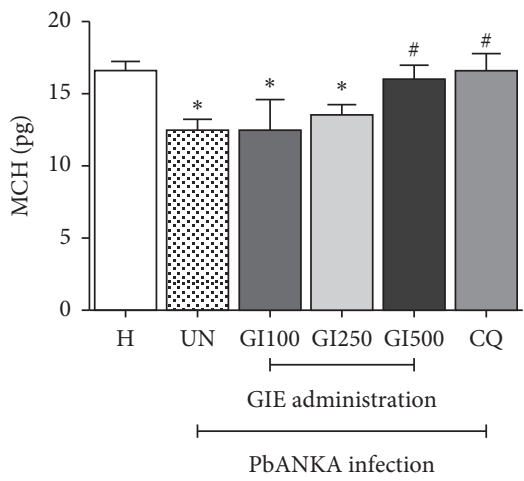

(e)

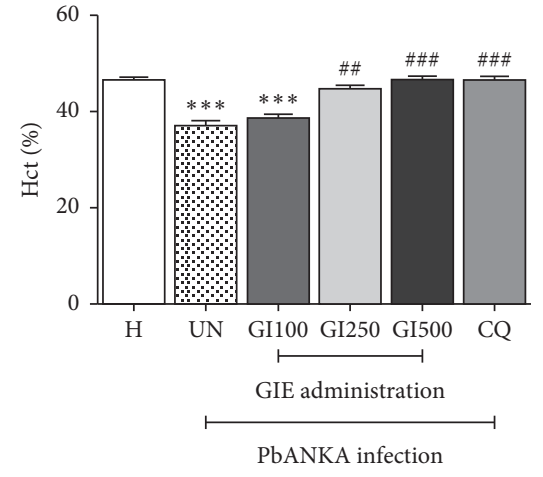

(c)

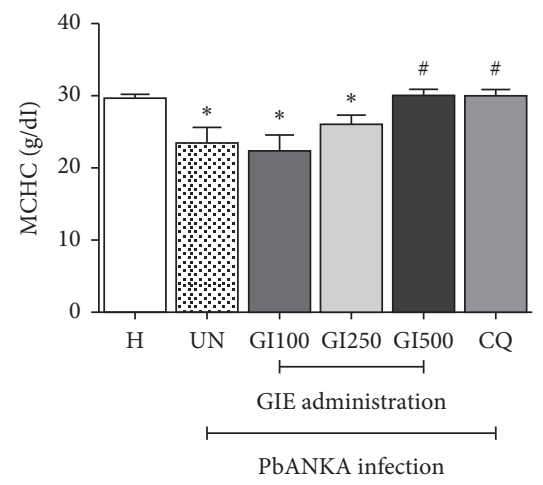

(f)

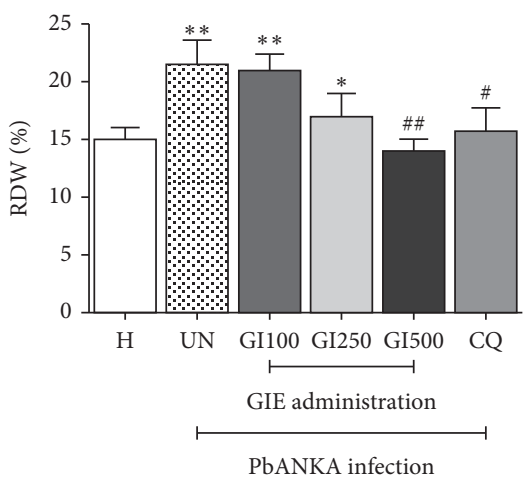

(g)

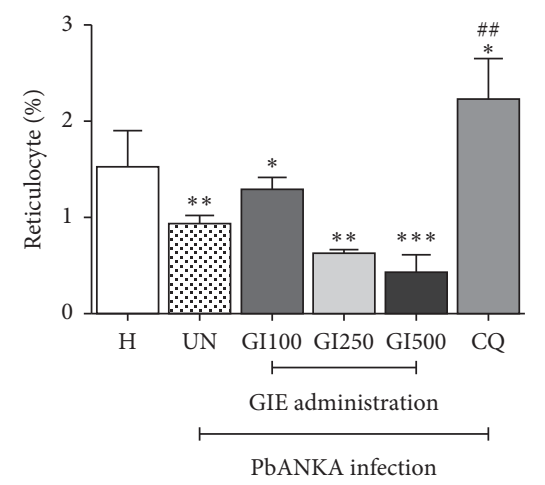

(h)

FIGURE 1: Effect of GIE on hematological alteration of RBC in PbANKA-infected mice. Groups of mice were infected intraperitoneally with $1 \times 10^{7}$ parasitized RBC of PbANKA and treated orally with 100,250 , and $500 \mathrm{mg} / \mathrm{kg}$ of GIE for 4 consecutive days. Hematological parameters of RBC were measured: (a) RBC count, (b) Hb, (c) Hct, (d) MCV, (e) MCH, (f) MCHC, (g) RDW, and (h) reticulocyte. $\mathrm{H}$ : healthy mice; UN: untreated mice; GI100, GI250, and GI500: 100, 250, and $500 \mathrm{mg} / \mathrm{kg}$ of GIE-treated mice, respectively; CQ: $10 \mathrm{mg} / \mathrm{kg}$ of CQ-treated mice. ${ }^{*} p<0.05,{ }^{* *} p<0.01$, and ${ }^{* * *} p<0.001$, compared with H. ${ }^{\#} p<0.05,{ }^{\# \#} p<0.01$, and ${ }^{\# \# \#} p<0.001$, compared with UN. Results were presented as mean + SEM.

from an increase in reticulocytes [25]. However, it appeared that the activity of the lower doses of GIE (100 and $250 \mathrm{mg}$ / $\mathrm{kg}$ ) was not strong enough to protect hemolysis and anemia during $\mathrm{PbANKA}$ infection in mice. This might be due to the low levels of active compounds in the extract whose activity could not be detected.

WBC plays a role in the immune defense against foreign antigens through the processes of leukocytosis and antibody production. Significantly increased WBC count, neutrophils, and monocytes and decreased lymphocytes in PbANKAinfected mice without treatment were observed. These results suggested that stimulation of the immune system by infection might be developed as a physiological response [26]. During malaria infection, phagocytosis activity by neutrophils and monocytes was first responded, while lymphocytes were still decreased [27]. Lymphocytes have an important role in the immune system to produce antibodies against malaria parasites after the phagocytic activity occurred [28]. Moreover, basophils and eosinophils were significantly increased in the untreated group, responsible for mediating inflammatory and cytotoxic events associated with malaria infection. Basophils play a crucial 


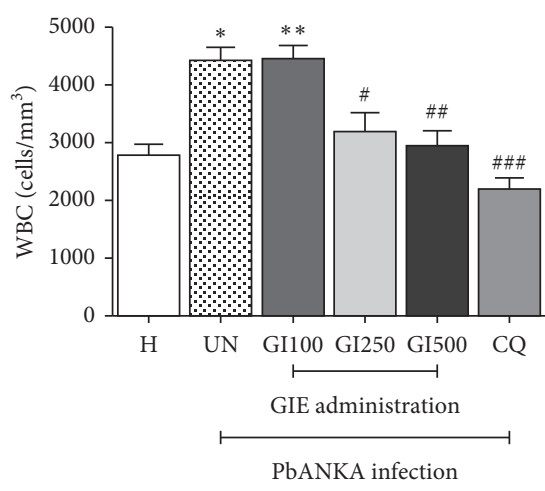

(a)

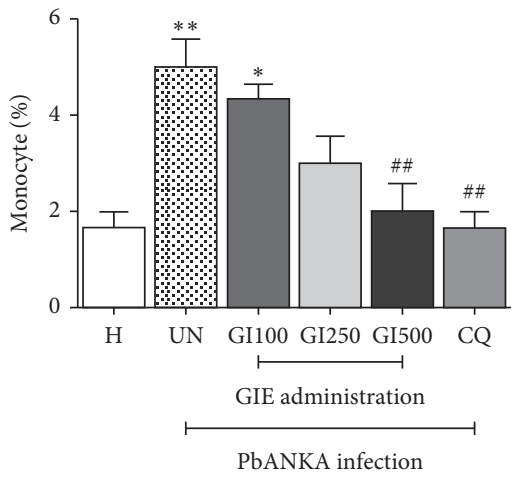

(d)

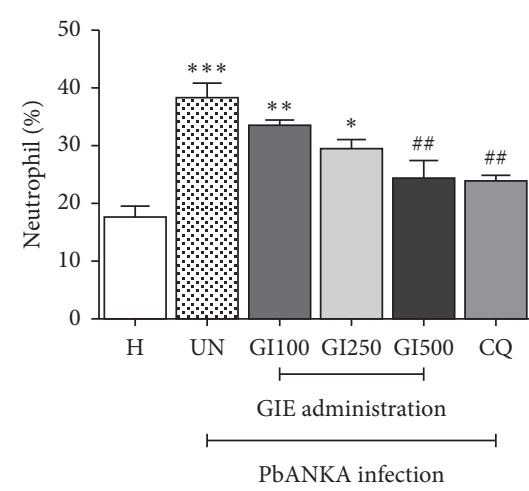

(b)

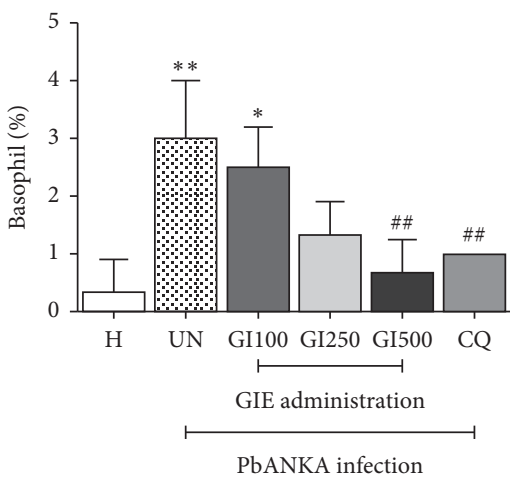

(e)

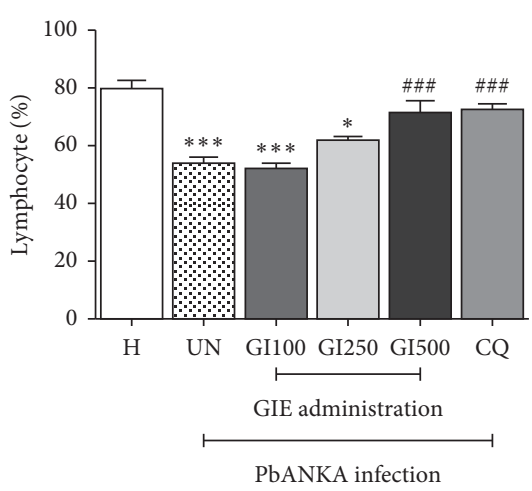

(c)

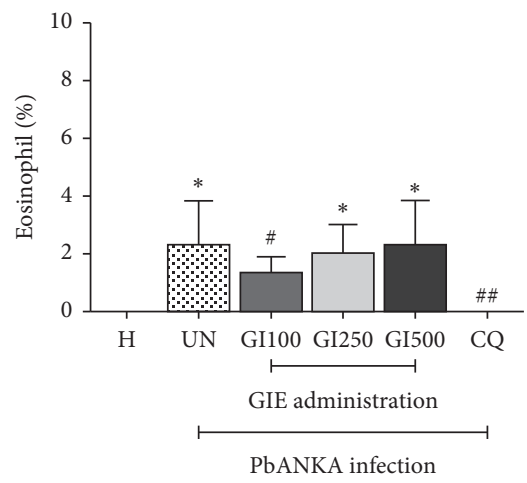

(f)

FIGURE 2: Effect of GIE on hematological alteration of WBC in PbANKA-infected mice. Groups of mice were infected intraperitoneally with $1 \times 10^{7}$ parasitized RBC of PbANKA and treated orally with 100,250 , and $500 \mathrm{mg} / \mathrm{kg}$ of GIE for 4 consecutive days. Hematological parameters of WBC were measured: (a) WBC count, (b) neutrophil, (c) lymphocyte, (d) monocyte, (e) basophil, and (f) eosinophil. H: healthy mice; UN: untreated mice; GI100, GI250, and GI500: 100, 250, and $500 \mathrm{mg} / \mathrm{kg}$ of GIE-treated mice, respectively, CQ: $10 \mathrm{mg} / \mathrm{kg}$ of CQ-treated mice. ${ }^{*} p<0.05,{ }^{* *} p<0.01$, and ${ }^{* * *} p<0.001$, compared with $\mathrm{H}$. ${ }^{\#} p<0.05,{ }^{\# \#} p<0.01$, and ${ }^{\# \# \#} p<0.001$, compared with UN. Results were presented as mean + SEM.

role in inflammatory reactions recruited to the sites of inflammation and drive proinflammatory responses [29, 30]. The present study, the ability of GIE to normalize the hematological parameters of WBC in the GIE-treated groups, especially at a dose of $500 \mathrm{mg} / \mathrm{kg}$, showed that the extract had immunomodulatory, antioxidant, and anti-inflammatory effects. These activities have been linked to the presence of polyphenols, flavonoids, alkaloids, terpenoids, tannin, and other phenolic compounds of the extracts consistent with GIE, which also contains these compounds [31]. Additionally, gymnemic acids, a major active compound presented in the GIE, might be considered to exert the protective effect on the alteration of hematological parameters induced by PbANKA infection in mice [32]. However, the active compounds responsible for the results in this study need to be further identified.

\section{Conclusion}

This study demonstrated that GIE has the potential effect on significantly protecting hematological alteration during $\mathrm{PbANKA}$ infection in mice. This effect might be attributed to active constituents in this extract, which may have acted singly or in synergy with others to show the activity observed in this study. However, the identification and characterization of the active compounds in the extract are warranted. Additionally, the active compounds should also be studied in clinical trials. Therefore, it justifies the use of GIE in the development of alternative agents for the management of malaria.

\section{Data Availability}

The data used to support the findings of this study are available at https://figshare.com/s/912935cb2e7d5370b478 (DOI: 10.6084/m9.figshare.14298872).

\section{Conflicts of Interest}

The authors declare that there are no conflicts of interest regarding the publication of this manuscript.

\section{Acknowledgments}

The authors would like to thank the B. Sc. students from the School of Allied Health Sciences, Walailak University, for supporting this study. The animal experiments and technical supports of the Research Institute for Health Sciences, 
Walailak University, are gratefully acknowledged. This study was partially funded by the Research Institute for Health Sciences, Chiang Mai University.

\section{References}

[1] N. J. White, S. Pukrittayakamee, T. T. Hien, M. A. Faiz, O. A. Mokuolu, and A. M. Dondorp, "Malaria," The Lancet, vol. 383, no. 9918, pp. 723-735, 2014.

[2] WHO, World Malaria Report, World Health Organization, Geneva, Switzerland, 2020, https://www.whoint/publications/ i/item/9789240015791.

[3] I. Ullah, M. U. Ali, S. Ali, A. Rafiq, Z. Sattar, and S. Hussain, "Hematological profile of patients having malaria-positive peripheral blood smears: a cross-sectional study at a diagnostic Research center in khyber pakhtunkhwa, Pakistan," Cureus, vol. 10, no. 9, Article ID e3376, 2018.

[4] S. Rojanasthien, V. Surakamolleart, S. Boonpucknavig, and P. Isarangkura, "Hematological and coagulation studies in malaria," Journal of Medical Association of Thailand, vol. 75, no. Suppl 1, pp. 190-194, 1992.

[5] N. J. White, "Does antimalarial mass drug administration increase or decrease the risk of resistance?" The Lancet Infectious Diseases, vol. 17, no. 1, pp. e15-e20, 2017.

[6] K. Tiamyom, K. Sirichaiwetchakoon, T. Hengpratom et al., "The effects of cordyceps sinensis (berk.) sacc. And Gymnema inodorum (lour.) Decne. Extracts on adipogenesis and lipase activity in vitro," Evidence-Based Complementary and Alternative Medicine, vol. 2019, Article ID 5370473, 13 pages, 2019.

[7] B. Dunkhunthod, C. Talabnin, M. Murphy, K. Thumanu, P. Sittisart, and G. Eumkeb, "Gymnema inodorum (lour.) Decne. Extract alleviates oxidative stress and inflammatory mediators produced by RAW264.7 macrophages," Oxidative Medicine and Cellular Longevity, vol. 2021, Article ID 8658314, 20 pages, 2021.

[8] W. Srinuanchai, R. Nooin, P. Pitchakarn et al., "Inhibitory effects of Gymnema inodorum (Lour.) Decne leaf extracts and its triterpene saponin on carbohydrate digestion and intestinal glucose absorption," Journal of Ethnopharmacology, vol. 266, Article ID 113398, 2021.

[9] K. Shimizu, M. Ozeki, K. Tanaka et al., "Suppression of glucose absorption by extracts from the leaves of Gymnema inodorum," Journal of Veterinary Medical Science, vol. 59, no. 9, pp. 753-757, 1997.

[10] L. A. Gaytán Martínez, L. A. Sánchez-Ruiz, L. Y. Zuñiga, M. González-Ortiz, and E. Martínez-Abundis, "Effect of Gymnema sylvestre administration on glycemic control, insulin secretion, and insulin sensitivity in patients with impaired glucose tolerance," Journal of Medicinal Food, vol. 24, no. 1 , pp. 28-32, 2021.

[11] V. P. Gaonkar and K. Hullatti, "Indian traditional medicinal plants as a source of potent Anti-diabetic agents: a Review," Journal of Diabetes and Metabolic Disorders, vol. 19, no. 2, pp. 1895-1908, 2020.

[12] G. Fabio, V. Romanucci, C. Marino, A. Pisanti, and A. Zarrelli, "Gymnema sylvestre R. Br., an Indian medicinal herb: traditional uses, chemical composition, and biological activity," Current Pharmaceutical Biotechnology, vol. 16, no. 6, pp. 506-516, 2015.

[13] F. Khan, M. M. R. Sarker, L. C. Ming et al., "Comprehensive review on phytochemicals, pharmacological and clinical potentials of Gymnema sylvestre," Frontiers in Pharmacology, vol. 10, p. 1223, 2019.
[14] W. Peters, J. H. Portus, and B. L. Robinson, "The chemotherapy of rodent malaria, XXII," Annals of Tropical Medicine and Parasitology, vol. 69, no. 2, pp. 155-171, 1975.

[15] T. Fushiki, A. Kojima, T. Imoto, K. Inoue, and E. Sugimoto, "An extract of Gymnema sylvestre leaves and purified gymnemic acid inhibits glucose-stimulated gastric inhibitory peptide secretion in rats," The Journal of Nutrition, vol. 122, no. 12, pp. 2367-2373, 1992.

[16] N. J. White, "Anaemia and malaria," Malaria Journal, vol. 17, no. 1 , p. $371,2018$.

[17] H. Haroon, P. A. Fazel, M. Naeem, A. Mobin, A. H. Naqvi, and K. Makki, "Hide and seek: hematological aspects of malaria - a developing country perspective," The Journal of Infection in Developing Countries, vol. 7, no. 03, pp. 273-279, 2013.

[18] G. S. Yap and M. M. Stevenson, "Inhibition of in vitro erythropoiesis by soluble mediators in Plasmodium chabaudi AS malaria: lack of a major role for interleukin 1, tumor necrosis factor alpha, and gamma interferon," Infection and Immunity, vol. 62, no. 2, pp. 357-362, 1994.

[19] J. McCullough, "RBCs as targets of infection," Hematology, vol. 2014, no. 1, pp. 404-409, 2014.

[20] G. A. Awandare, J. J. Martinson, T. Were et al., "MIF (Macrophage migration inhibitory factor) promoter polymorphisms and susceptibility to severe malarial anemia," The Journal of Infectious Diseases, vol. 200, no. 4, pp. 629-637, 2009.

[21] G. A. Awandare, P. Kempaiah, D. O. Ochiel, P. Piazza, C. C. Keller, and D. J. Perkins, "Mechanisms of erythropoiesis inhibition by malarial pigment and malaria-induced proinflammatory mediators in an in vitro model," American Journal of Hematology, vol. 86, no. 2, pp. 155-162, 2011.

[22] O. A. Skorokhod, L. Caione, T. Marrocco et al., "Inhibition of erythropoiesis in malaria anemia: role of hemozoin and hemozoin-generated 4-hydroxynonenal," Blood, vol. 116, no. 20, pp. 4328-4337, 2010.

[23] L. Xu, X. Zheng, K. Berzins, and A. Chaudhuri, "Cytokine dysregulation associated with malarial anemia in Plasmodium yoelii infected mice," American Journal of Translational Research, vol. 5, no. 2, pp. 235-245, 2013.

[24] L. C. Mourão, P. M. d. S. Roma, J. d. S. Sultane Aboobacar et al., "Anti-erythrocyte antibodies may contribute to anaemia in Plasmodium vivax malaria by decreasing red blood cell deformability and increasing erythrophagocytosis," Malaria Journal, vol. 15, no. 1, p. 397, 2016.

[25] A. Ballal, A. Saeed, P. Rouina, and W. Jelkmann, "Effects of chloroquine treatment on circulating erythropoietin and inflammatory cytokines in acute Plasmodium falciparum malaria," Annals of Hematology, vol. 88, no. 5, pp. 411-415, 2009.

[26] C. Jerusalem and W. Kretschmar, "Changes in the leukocyte picture in malaria (Plasmodium berghei) in NMRI mice and their significance for the course of the infection," $Z$ Tropenmed Parasitol, vol. 16, pp. 235-257, 1965.

[27] G. Kapoor, U. Bagai, and H. S. Banyal, "Plasmodium berghei induces apoptotic changes in splenic and peripheral blood cells," Tropical Biomedicine, vol. 28, no. 1, pp. 119-124, 2011.

[28] A. A. Omonkhua, M. C. Cyril-Olutayo, O. M. Akanbi, and O. A. Adebayo, "Antimalarial, hematological, and antioxidant effects of methanolic extract of Terminalia avicennioides in Plasmodium berghei-infected mice," Parasitology Research, vol. 112, no. 10, pp. 3497-3503, 2013.

[29] W. Beghdadi, A. Porcherie, B. S. Schneider et al., "Rôle de l'histamine et des récepteurs histaminiques dans la 
pathogenèse du paludisme," Médecine/Sciences, vol. 25, no. 4, pp. 377-381, 2009.

[30] S. Pelleau, S. Diop, M. Dia Badiane et al., "Enhanced basophil reactivities during severe malaria and their relationship with the Plasmodium falciparum histamine-releasing factor translationally controlled tumor protein," Infection and Immunity, vol. 80, no. 8, pp. 2963-2970, 2012.

[31] A. I. Calderón, J. Simithy-Williams, and M. P. Gupta, "Antimalarial natural products drug discovery in Panama," Pharmaceutical Biology, vol. 50, no. 1, pp. 61-71, 2012.

[32] M.-H. Kang, M. S. Lee, M.-K. Choi, K.-S. Min, and T. Shibamoto, "Hypoglycemic activity of Gymnema sylvestre extracts on oxidative stress and antioxidant status in diabetic rats," Journal of Agricultural and Food Chemistry, vol. 60, no. 10, pp. 2517-2524, 2012. 